S. Matsumoto and A. Sato

Nagoya Math. J.

Vol. 122 (1991), 75-82

\title{
PERTURBATION OF THE HOPF FLOW AND TRANSVERSE FOLIATIONS
}

\author{
SHIGENORI MATSUMOTO AND ATSUSHI SATO \\ Dedicated to Professor Ken'ichi Shiraiwa on his 60th birthday
}

\section{$\S 1$. Introduction}

Consider a nonsingular vector field $X$ on a closed manifold $M^{n}$. As a matter of fact, $X$ always admits a transverse codimension one plane field, which however may fail to be integrable. In fact it is well known that there are many examples of vector fields which do not admit transverse foliations.

The purpose of this paper is to contribute to the study of the topology of the set of vector fields which admit transverse foliations. In what follows, we only consider the three dimensional case, since there the qualitative study of foliations is a rather mature stage and provides us with powerful tools. For example, a complete criterion for admitting transverse foliations is obtained for 3-dimensional Smale flows by Goodman ([G1], [G2]).

Denote by $N S\left(M^{3}\right)$ the set of nonsingular $C^{r}$ verctor fields $(1 \leq r$ $<\infty)$ on a 3-manifold $M^{3}$ and by $\pitchfork\left(M^{3}\right)$ the subset of those vector fields which admit transverse codimension one $C^{1}$ foliations. Andrade ([A]), working with the $C^{0}$ topology on $N S\left(M^{3}\right)$, has shown that for any 3manifold $M^{3}$,

$$
\pitchfork\left(M^{3}\right) \varsubsetneqq \operatorname{Int} \overline{\pitchfork\left(M^{3}\right)},
$$

where he made use of Goodman's criterion and the fact that Smale flows are $C^{0}$ dense in $N S\left(M^{3}\right)$.

Our theme here is to consider the same problem in the context of the $C^{r}$ topology. Henceforth in this paper we assume that the set $N S\left(M^{3}\right)$ of nonsingular $C^{r}$ vector fields is equipped with the $C^{r}$ topology $(1 \leq r$ $<\infty)$. Obviously $\pitchfork\left(M^{3}\right)$ is an open subset of $N S\left(M^{3}\right)$.

Received February 2, 1990. 
Let $H \in N S\left(S^{3}\right)$ be the Hopf vector field, that is, the one associated with the principal $S^{1}$ bundle $S^{1} \rightarrow S^{3} \rightarrow S^{2}$. As is well known, $H \notin \pitchfork\left(S^{3}\right)$. In fact this is shown as follows. Suppose on the contrary that $H$ admits a transverse foliation $\mathscr{F}$. Then by Novikov compact leaf theorem, $\mathscr{F}$ has a toral leaf $L$. But the orbit of $H$ which passes through $L$ must be nonclosed. A contradiction.

The following is our main result.

THEOREM (1.1).

$$
H \in \operatorname{Int} \overline{\pitchfork\left(S^{3}\right) \backslash \pitchfork\left(S^{3}\right) .}
$$

Theorem (1.1) is inspired by a question raised by Andrade ([A]). He asked whether or not $H$ belongs to the difference set in the $C^{0}$ topology. However notice that our result (1.1), in the $C^{r}$ topology, has no connection with his original question. We suspect that $H \notin \operatorname{Int} \overline{\pitchfork\left(S^{3}\right)}$ in the $C^{0}$ topology.

Let us remark that $H$ is not an isolated point in $N S\left(S^{3}\right) \backslash \pitchfork\left(S^{3}\right)$. In fact it is easy to construct an infinite sequence $\left\{X_{j}\right\}$ of points of $N S\left(S^{3}\right) \backslash$ $\pitchfork\left(S^{3}\right)$ which converges to $H$ in the $C^{\infty}$ topology.

Here is an outline of the proof of (1.1). Our purpose is to show that $\pitchfork\left(S^{3}\right)$ is dense in some small neighborhood of $H$. In $\S 2$, we prepare two basic theorems; one Seifert closing theorem and the other KupkaSmale density theorem. They allow us to consider only such a perturbation $X$ of $H$ which has a great circle $C \subset S^{3}$ as a hyperbolic closed orbit. In $\S 3$, we construct a Birkhoff section $B$ for $X$ such that $\partial B=C$. When $C$ is attracting or repelling, this immediately allows us to construct a transverse foliation. When $C$ is a saddle, the study of the first return map of $B$ in $\S 4$ shows the existence of another great circle orbit which is either attracting or repelling. Hence this case is reduced to the former case.

\section{§ 2. Preparations}

Let $X$ be a small $C^{0}$ perturbation of $H$ and let $\gamma$ be closed orbit of $X$.

Definition (2.1). $\quad \gamma$ is called a short closed orbit in case there exists a solid torus $A \subset S^{3}$ such that

(a) $A$ is a union of orbits of $H$, 
(b) $r \subset \operatorname{Int} A$,

(c) $r$ is homotopic in $A$ to the longitude.

In 1950 paper ([Se]), Seifert has shown the existence of a closed orbit for a perturbation of the Hopf vector field $H$. Furthermore one knows by the proof that what he found is in fact a short closed orbit.

THEOREm (2.2). If $X$ is sufficiently $C^{0}$ near $H$, then $X$ admits a short closed orbit.

Next we prepare Kupka-Smale density theorem ([K, $[\mathrm{Sm}])$. However all that we need is the following "truncated" version. For a neat treatment, see Peixoto ([P]).

Definition (2.3). A $C^{r}$ nonsingular vector field $X$ on a closed manifold $M^{n}$ is called T-hyperbolic $(T>0)$, if all the closed orbits of $X$ of period $\leq T$ are hyperbolic.

THEOREM (2.4). T-hyperbolic vector fields form an open dense subset in $N S\left(M^{3}\right)$ for any $T>0$.

Now let us embark upon the proof of Theorem (1.1). We look upon $S^{3}$ as the unit sphere in the complex plane $\mathbf{C}^{2}$. The flow $\left\{h_{t}\right\}$ of $H$ is given by

$$
h_{t}(u, v)=\left(e^{2 \pi i t} u, e^{2 \pi i t} v\right) .
$$

Thus all the orbits of $H$ are great circles of $S^{3}$ and have period 1 .

Consider a small $C^{r}$ neighborhood $\mathscr{U}$ of $H$. By Theorem (2.4), 3hyperbolic vector fields are dense in $\mathscr{U}$. Take such $Y$ and let us show that $Y \in \pitchfork\left(S^{3}\right)$. By Theorem (2.2), $Y$ has a short closed orbit $\gamma$. Clearly $\gamma$ is $C^{r+1}$ close to some closed orbit $C$ of $H$ (a great circle). Therefore there exists a $C^{r+1}$ diffeomorphism $f$ of $S^{3}, C^{r+1}$ close to the identity, which carries $\gamma$ to $C$. Thus if we choose $Y$ from an even smaller neighborhood, we have that $X=f_{*} Y \in \mathscr{U}$ and that $X$ is 2-hyperbolic.

Now Theorem (1.1) reduces to the following proposition.

Proposition (2.5). Suppose

(i) $X \in N S\left(S^{3}\right)$ is sufficiently $C^{r}$ close to $H$,

(ii) $X$ has a great circle orbit $C$,

(iii) $X$ is 2-hyperbolic.

Then $X$ admits a codimension one transverse smooth foliation. 


\section{§3. Birkhoff section}

Let $X$ be a vector field satisfying the conditions of Proposition (2.5). Assume for simplicity that $X$ has the great circle

$$
C=\{v=0\} \subset S^{3}=\left\{(u, v) \in \mathbf{C}^{2} ;|u|^{2}+|v|^{2}=1\right\}
$$

as closed orbit. Let

$$
A=\{|v|<1 / \sqrt{2}\} .
$$

$A$ is a solid torus in $S^{3}$ containing $C$ as its center. A point of $A$ is indicated by

$$
(x, y ; \tau) \in D^{2} \times(\mathbf{R} / 2 \pi \mathbf{Z}),
$$

where $\sqrt{2} v=x+i y$ and $\tau=\arg u$. Using this coordinates, the Hopf vector field is given by

$$
H=-y \frac{\partial}{\partial x}+x \frac{\partial}{\partial y}+\frac{\partial}{\partial \tau} .
$$

Put

$$
X=f \frac{\partial}{\partial x}+g \frac{\partial}{\partial y}+k \frac{\partial}{\partial \tau},
$$

where $f, g$ and $k$ are $C^{r}$ functions on $A$. By conditions (i) and (ii) of (2.5), they satisfy for some small $\varepsilon>0$,

$$
\left\{\begin{array}{l}
f(0,0 ; \tau)=g(0,0 ; \tau)=0 \\
\|k-1\|<\varepsilon \\
\left\|f_{x}\right\|<\varepsilon,\left\|f_{y}+1\right\|<\varepsilon,\left\|f_{\tau}\right\|<\varepsilon \\
\left\|g_{x}-1\right\|<\varepsilon,\left\|g_{y}\right\|<\varepsilon,\left\|g_{\tau}\right\|<\varepsilon
\end{array}\right.
$$

where $\|\cdot\|$ denotes the sup norm on $A$.

Let $(r, \theta)$ be the polar coordinates of $(x, y)$. Computing the derivative $\dot{\theta}$ along the integral curve of $X$, one gets

$$
\dot{\theta}=\frac{1}{r}(-f \sin \theta+g \cos \theta) .
$$

Notice that $-f \sin \theta+g \cos \theta$ is a differentiable function of $r$ if one fixes the value of $\theta$. Therefore by the mean value theorem, we have 
(4)

$$
\begin{aligned}
\dot{\theta} & =\left.\frac{\partial}{\partial r}(f \sin \theta+g \cos \theta)\right|_{r=r^{\prime}} \\
& =-\left(f_{x} \cos \theta+f_{y} \sin \theta\right) \sin \theta+\left(g_{x} \cos \theta+g_{y} \sin \theta\right) \cos \theta \\
& \geq(1-\varepsilon)\left(\sin ^{2} \theta+\cos ^{2} \theta\right)-2 \varepsilon|\cos \theta \sin \theta| \\
& \geq 1-2 \varepsilon .
\end{aligned}
$$

Likewise we have

$$
\dot{\theta} \leq 1+2 \varepsilon .
$$

Let

$$
B=\{v \geq 0\} \subset S^{3} .
$$

$B$ is a closed disc. $B \cap A$ is given by the equality $\theta=0$. We claim that $B$ is a "Birkhoff section" for $X$. Denote by $\left\{\varphi_{t}\right\}$ the flow generated by $X$.

Proposition (3.1).

(a) $\partial B=C$.

(b) Int $B$ is transverse to $X$.

(c) For any $x \in S^{3}$, one has $\varphi_{t}(x) \in B$ for some $0<t \leq 1 /(1-2 \varepsilon)$.

Proof. (a) is clear. (b) and (c) are immediate consequence of (4) for orbits lying in $A$. For other orbits, they are also obvious by the $C^{0}$ closeness of $X$ to $H$.

Q.E.D.

\section{$\S 4$. Construction of a foliation}

Notice that $C=\partial B$ is a hyperbolic closed orbit by (ii) and (iii) of (2.5).

Case 1. $C$ is an attractor (or a repeller). Consider the singular foliation by discs $\mathscr{G}=\left\{B_{\theta}\right\}_{0 \leq \theta<2 \pi}$, where

$$
B_{\theta}=\{\arg v=\theta\} \text {. }
$$

Clearly $X$ is transverse to $\mathscr{G}$ except the singular locus $C$ of $\mathscr{G}$. Let us modify $\mathscr{G}$ to a transverse foliation.

First of all by a standard argument, one can find a small tubular neighborhood $W$ of $C$ such that $X$ is transverse to $\partial W$. Turbulize $\mathscr{G}$ around $\partial W$ in such a way that the normal orientation is compatible with the flow orientation on each leaf. Then we obtain a foliation outside $W$ which is transverse to $X$. Next put a Reeb component inside $W$ in a 

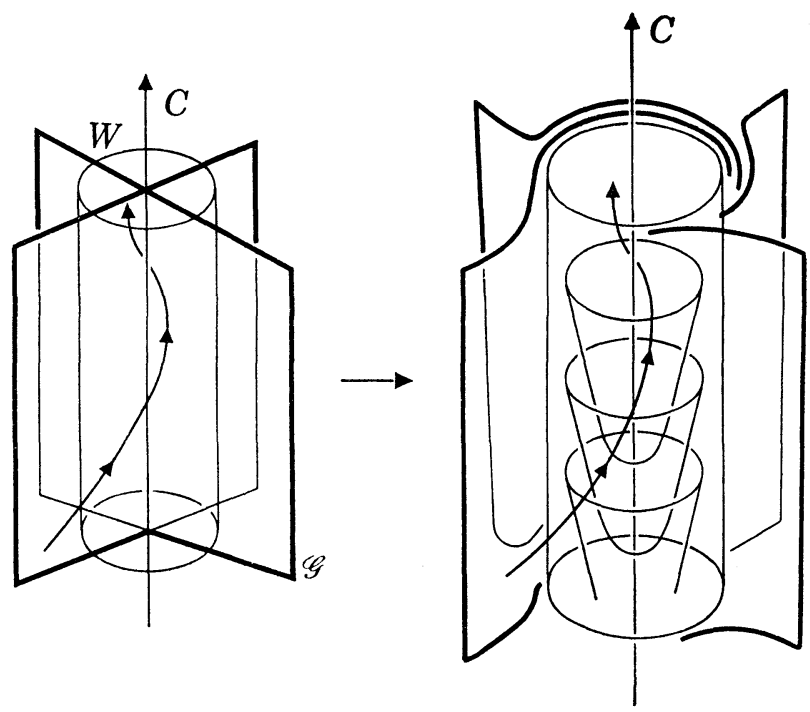

Figure 1.

suitable direction so that it is transverse to $X$. This completes the construction of a transverse foliation on $S^{3}$. See Figure 1 .

Case 2. $C$ is a saddle. Let $\psi$ : Int $B \rightarrow \operatorname{Int} B$ be the first return map of the flow $X$.

Lemma (4.1). $\psi$ extends to a homeomorphism of $B$ such that $\psi \mid \partial B$ has two attractors and two repellers.

Proof. Define a mapping

$$
\alpha: \mathscr{A}=[0,1] \times(\mathbf{R} / 2 \pi \mathbf{Z}) \times(\mathbf{R} / 2 \pi \mathbf{Z}) \longrightarrow A
$$

by $\alpha(r, \theta ; \tau)=(r \cos \theta, r \sin \theta ; \tau) . \quad \alpha$ is a diffeomorphism on $\mathscr{A}^{0}=p_{1}^{-1}((0,1])$, where $p_{1}$ is the projection onto the first factor. Denote the torus $\mathscr{A} \backslash \mathscr{A}^{0}$ by $\mathscr{T}$. Define the vector field $\tilde{X}$ on $\mathscr{A}$ by

$$
\begin{cases}\tilde{X}=\alpha_{*}^{-1} X & \text { on } \mathscr{A}^{0} \\ \tilde{X}(0, \theta ; \tau)=\frac{\partial}{\partial r}(-f \sin \theta+g \cos \theta) \frac{\partial}{\partial \theta}+k \frac{\partial}{\partial \tau} & \text { on } \mathscr{T},\end{cases}
$$

where as before $X$ is given by (1). By (3), $\tilde{X}$ is continuous and by (4) and (5), it is $C^{0}$ close to $\partial / \partial \theta+\partial / \partial \tau$ on $\mathscr{T}$. Define

$$
\mathscr{B}=\{\theta=0\} \subset \mathscr{A} \text {. }
$$


$\mathscr{B}$ is the pull back image of $B$ by $\alpha$. Clearly the first return map (FRM) of $\mathscr{B}$ for $\tilde{X}$ is conjugate by $\alpha$ to the FRM $\psi$ of Lemma (4.1). This shows that $\psi$ extends to the whole of $B$ and $\psi \mid \partial B$ coincides with the FRM $\tilde{\psi}$ of the cross section $\mathscr{B} \cap \mathscr{T}$ for $\tilde{X} \mid \mathscr{T}$. Now there is another cross section $\mathscr{D}=\{\tau=0\} \subset \mathscr{T}$. The FRM $\tilde{\eta}$ of $\mathscr{D}$ is of course related with the FRM $\eta$ for $X$ at the corresponding meridian $\operatorname{disc} D$ of A. In fact $\tilde{\eta}$ is computed in terms of the first derivative of $\eta$ at the origin. Since $C$ is a saddle, $\tilde{\eta}$ has two attractors and two repellers. Now it is easy to show that $\tilde{\psi}$ is conjugate to $\tilde{\eta}$. This shows the lemma.

Q.E.D.

Let $W^{u}$ be the unstable manifold of $C$. $W^{u}$ winds once around $C$ and cut $B$ near $C$ in $\operatorname{arcs} a_{1}$ and $a_{2}$ which are transverse to $C$. See Figure 2. Likewise the stable manifold $W^{s}$ cuts $B$ in $\operatorname{arcs} b_{1}$ and $b_{2}$ transverse to $C$. The behavior of $\psi$ near $C$ is shown in Figure 3.

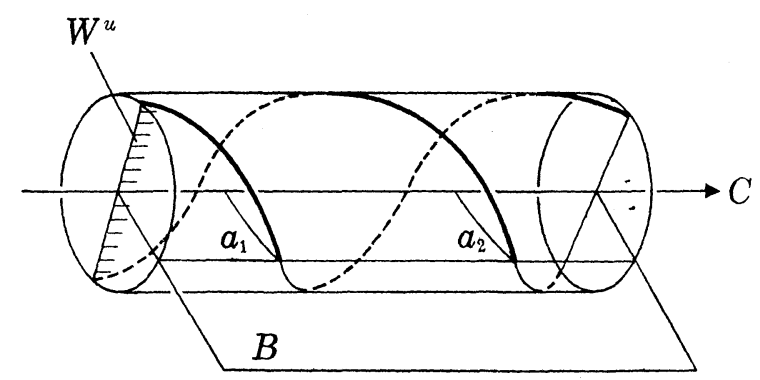

Figure 2 .

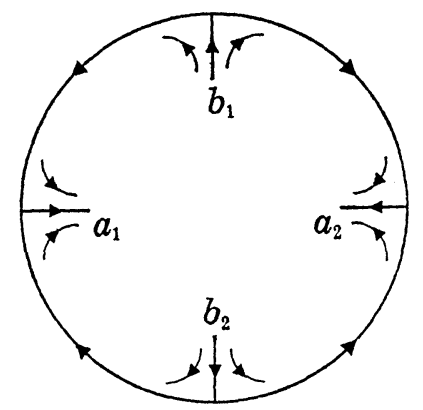

Figure 3.

Consider the double $D \psi$ of $\psi$ :

$$
D \psi: D B \longrightarrow D B \text {. }
$$

$D B$ is homeomorphic to the 2-sphere and since $X$ is a 2-hyperbolic vector 
field and by (3.1) (c), the first return time of $\psi$ is less than $1 /(1-2 \varepsilon)$, all the fixed points of $\psi$ are hyperbolic. $\psi$ has already four fixed points on $\partial B$, each with the Lefschetz index -1 . Therefore by the index theorem, there exists an attractor or a repeller, which corresponds to a short closed orbit. Now Case 2 is reduced to Case 1. Detailed arguments are left to the reader.

\section{REFERENCES}

[A] P. F. A. Andrade, Fluxos que admitem folheações transversais, Thesis, P. U. C., Rio de Janeiro 1984.

[G1] S. Goodman, Vector fields with transverse foliations, Topology, 24 (1985), 333340 .

[G2] — Vector fields with transverse foliations II, Ergodic Theory and Dynamical Systems, 6 (1986), 193-203.

[K] I. Kupka, Contribution à la théorie des champs générique, Contrib. Differential Equations, 2 (1963), 457-482.

[P] M. M. Peixoto, On an approximation theorem of Kupka and Smale, J. Differential Equations, 3 (1966), 214-227.

[Se] H. Seifert, Closed integral curves in 3-space and isotopic two-dimensional deformations, Proc. Amer. Math. Soc., 1 (1950), 287-302.

[Sm] S. Smale, Stable manifolds for differential equations and diffeomorphisms, Ann. Scuola Norm. Sup. Pisa, 17 (1963), 97-116.

S. Matsumoto

Department of Mathematics

College of Science and Technology

Nihon University

1-8 Kanda-Surugadai

Chiyoda Ward

Tokyo 101 Japan

A. Sato

Department of Mathematics

School of Science and Technology

Meiji University

1-1-1 Higashi-Mita

Tama Ward, Kawasaki

Kanagawa 214 Japan 